\title{
An avian muscle factor related to MyoD1 activates muscle-specific promoters in nonmuscle cells of different germ-layer origin and in BrdU-treated myoblasts
}

\author{
Ze-yu Lin, Claude A. Dechesne, Juanita Eldridge, and Bruce M. Paterson \\ Laboratory of Biochemistry, National Cancer Institute, National Institutes of Health, Bethesda, Maryland 20892 USA
}

\begin{abstract}
We isolated the cDNA encoding a myogenic factor expressed in embryonic chick breast muscle by virtue of its weak hybridization to the mouse MyoD1 clone. Nucleotide sequence analysis and amino acid comparison define this clone, CMD1, as encoding a protein similar to mouse MyoD1. CMD1 encodes a polypeptide smaller than MyoD1, 298 versus 318 amino acids, respectively, and is $80 \%$ concordant by amino acid sequence overall. The basic and myc domains required for myogenic conversion of mouse $10 \mathrm{~T}^{1 / 2}$ 'fibroblasts' to myoblasts with MyoD1 are completely conserved in CMD1. CMD1 is just as efficient as the mouse homolog in myogenic conversion of $10 \mathrm{~T}^{1 / 2}$ cells and coactivates the endogenous mouse MyoD1 gene in the process. The efficiency of myoblast conversion depends on the levels of CMD1 expression and suggests that the cellular concentration of CMD1 plays a role in the onset of myogenesis. Transient expression of CMD1 in a variety of nonmuscle cells from different germ-layer origins activates both cotransfected muscle-specific promoters and, in some cases, endogenous muscle-specific genes. 5-Bromodeoxyuridine (BrdU) treatment of chicken and mouse myoblasts reduces the expression of CMD1 and MyoD1, respectively, and may explain how this thymidine analog inhibits myogenesis and the activity of transfected muscle-specific promoters in BrdU-treated myoblasts. Transient expression of CMD1 in BrdU-treated myoblasts reactivates cotransfected muscle-specific promoters. CMD1 activates muscle-specific promoters in cotransfections regardless of cell type, whereas 'housekeeping' or constitutive promoters can be activated moderately, unaffected, or repressed, depending on the promoter and cell background. The rate and degree of myogenic conversion may be more restricted by cell phenotype than by germ-layer origin.
\end{abstract}

[Key Words: MyoD1; CDM1; myogenin; 10T1/2 cells]

Received February 28, 1989; revised version accepted May 4, 1989.

The original observation of Taylor and Jones (1979), demonstrating that treatment of $\mathrm{C} 3 \mathrm{H} 10 \mathrm{~T}^{1} / 2$ mouse embryonic fibroblasts with 5-azacytidine converted this cell into three distinct mesodermal cell types that represent muscle, cartilage, and fat tissues, provided a system in which to study the molecular basis of cellular determination. The stable and heritable nature of the various phenotypes, coupled with the high frequency of myogenic conversion suggested that only one or several genes were involved in converting the parental $10 \mathrm{~T}^{1} / 2 \mathrm{fi}$ broblast into a stable myoblast (Konieczny and Emerson 1984). Transfection experiments with DNA from either the 5-azacytidine-derived or $\mathrm{C} 2 \mathrm{Cl} 2$ myoblast, but not from the $10 \mathrm{~T}^{1} / 2$ fibroblast, converted $10 \mathrm{~T}^{1} 1 / 2$ fibroblasts to myoblasts with a frequency that suggested a single gene was involved (Lassar et al. 1986). Following these observations Lassar and associates (Davis et al. 1987) isolated a single cDNA clone, MyoD1, representing a gene product expressed uniquely in myogenic cells, that was capable of converting a variety of mesodermal fibroblasts into myoblasts when expressed in these cells. Human genomic DNA cloned into a cosmid vector could also induce myogenic conversion of $10 \mathrm{~T} 1 / 2$ cells, and this gene did not appear to correspond to MyoDl by Southern analysis (Pinney et al. 1988) yet could activate the endogenous MyoDl gene. Different myogenic factors, myogenin and myf5, recently have been isolated from rat (Wright et al. 1989) and human (Braun et al. 1989|, respectively, that are related functionally to but distinct from MyoD. Together, these results suggested that myogenic conversion may involve some type of hierarchy or network of myogenic determination genes. We have taken the mouse MyoDl clone to isolate myogenic factors involved in avian myogenesis.

Taking advantage of the weak cross-hybridization to mouse MyoD1, we isolated a cDNA clone encoding a closely related myogenic factor expressed in embryonic chick skeletal muscle, CMD1 /chicken myogenic deter- 
mination factor 1). Expression of this clone under the control of the Rous sarcoma virus (RSV) or SV40 viral promoters activates cotransfected muscle-specific promoters joined to the CAT reporter gene in differentiated cells from nonmuscle tissues and in various cell types representing all of the embryonic germ-layers. Efficient myoblast conversion of $10 \mathrm{~T}^{1 / 2}$ cells depends on the level of CMD1 expression, and this can be controlled by the strength of the viral promoter regulating expression. CMD1 supplied in trans can activate muscle-specific promoters in 5-bromodeoxyuridine (BrdU)-treated myoblasts, suggesting a mechanism for BrdU inhibition of myogenesis. Sequence and structural comparisons indicate that CMDl is the avian counterpart to mouse MyoD1 and is encoded by a single gene, as determined by Southern blot analysis.

\section{Results}

\section{Isolation of the avian cDNA clone CMD1}

Using hybridization conditions of reduced stringency, we screened a chicken cDNA library in $\lambda$ gt 10 prepared from well-fused cultures of embryonic chick breast muscle with the $1.8-\mathrm{kb}$ MyoD1 insert. Five cDNA clones were plaque purified from $\sim 2 \times 10^{6}$ recombinant phage. Two different insert patterns were obtained with either a single $E c o$ RI insert of $1.5 \mathrm{~kb}$ or three EcoRI inserts totaling $4.5 \mathrm{~kb}$. The smallest insert in the latter instance, the only hybridizing fragment, was identical in size to the insert in the former clone; therefore, the entire nucleotide sequence of the $1.5-\mathrm{kb}$ fragment was determined. The sequence of the 1518-nucleotide insert, called $\mathrm{CMDl}$, and the longest open reading frame $(\mathrm{ORF})$ of 298 amino acids are shown in Figure 1. The ORF encodes a polypeptide of 36,967 daltons and is 20 amino acids shorter than the MyoDl ORF. The first AUG is 155 nucleotides, $3^{\prime}$ to the start of the clone, and does not represent a particularly good vertebrate translational initiation consensus sequence: An $\mathrm{A}$ rather than a $\mathrm{C}$ residue at the -3 position is preferred. Similarly, the MyoDl consensus (Davis et al. 1987) has a G in the -3 position, which is only slightly preferable to a $\mathrm{C}$ nucleotide (Cavener 1987). Expression of both CMD1 and MyoDl polypeptides in reticulocyte lysates, using mRNAs produced with the SP6 bacterial promoter joined to the cDNA clones, produced methionine-labeled polypeptides of $\sim 45,000$ daltons (SDS-PAGE) suggesting that the first methionine is the initiator methionine (data not shown). A similar size discrepancy between the observed and actual molecular weight of CMD1 and MyoD1 also has been noted for the yeast activator protein GCN4 (Hope and Struhl 1985). The 3'noncoding region after the termination codon is $468 \mathrm{nu}-$ cleotides long, shorter than the 3 '-untranslated region of MyoDl. A putative adenylation consensus is apparent at nucleotide 1262, but it has not been verified experimentally. The additional sequence $3^{\prime}$ to this single putative adenylation signal may indicate that there is more than one adenylation site in the transcript, as seen with the

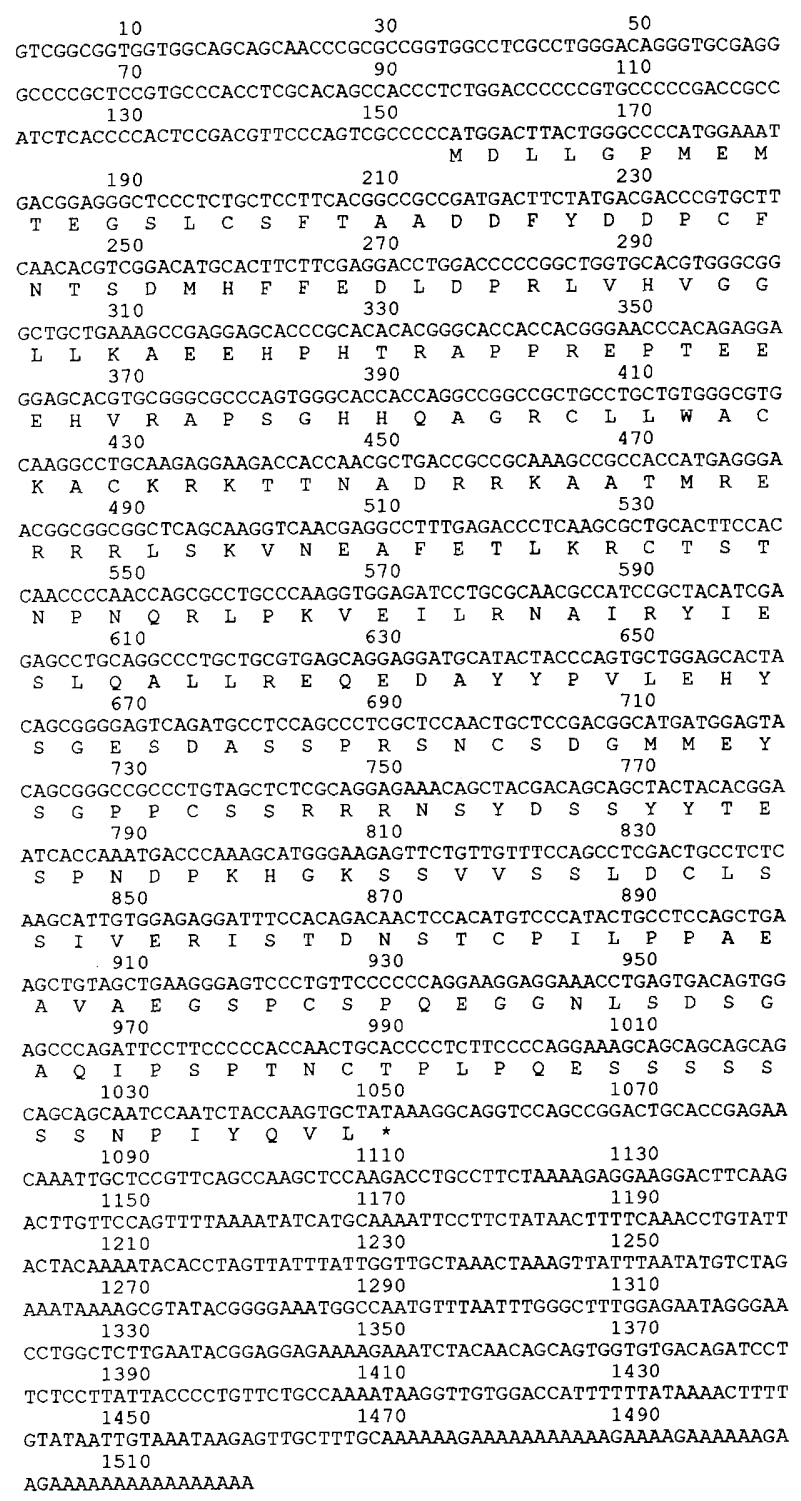

Figure 1. Nucleotide sequence and amino acid sequence of the longest ORF in the avian CMD1 cDNA clone. Note the canonical poly(A) addition signal at 1262 .

chicken vimentin gene (Zehner and Paterson 1983), and that the CMD1 mRNA transcript is slightly longer than indicated by the cDNA clone.

Northern analysis of CMD1 expression in various tissues of the chicken and in cultures of embryonic chick skeletal muscle indicated that the $1.5-\mathrm{kb}$ transcript of CMD1 was expressed only in skeletal muscle and not in brain, liver, heart, and lymphocytes (data not shown). Analysis of poly(A)-selected RNA from wellfused breast muscle cultures did show, in addition to the major $1.5-\mathrm{kb}$ transcript, a minor transcript of $\sim 1.2 \mathrm{~kb}$ that may represent the use of the $5^{\prime}$ adenylation signal, but this has not been determined experimentally. Southern analysis with genomic chicken DNA digested with HindIII, BamHI, and EcoRI indicated that the 
CMD1 gene is single copy, as only a single hybridizing band was observed in each digest (data not shown).

The basic and myc structural domains are conserved highly in the avian CMD1 polypeptide

Upon direct comparison of the amino acid sequences of CMD1 and MyoD1 (Fig. 2), using MyoDl as the amino acid position reference, several regions of sequence conservation were readily apparent and included the acidic amino terminus up to amino acid 61 , a histidine-cysteine cluster between amino acids 62 and 101, a highly basic region from amino acid 102 to 124 , a region with strong homology to the myc family of proteins between amino acid 141 and 162, and a serine-rich stretch of amino acids between positions 190 and 224 . The most striking feature was the highly conserved stretch of nearly 100 amino acids between positions 77 and 170 , containing the basic and myc homology regions that have been shown to be all that is required for myogenic conversion of $10 \mathrm{~T}^{1 / 2} 2$ cells (Tapscott et al. 1988). Overall, there is an $80 \%$ concordance between the amino acid sequences of CMD1 and MyoDl: Excluding the basicmyc domain (amino acids 93-153 in CMD1), which shows almost perfect identity, CMDl and MyoDl are $\sim 78 \%$ homologous on the amino-terminal side of the basic-myc domain and $67 \%$ homologous in the carboxyterminal portion. This compares to $13 \%$ and $36 \%$ homology, respectively, for Myf5 (Braun et al. 1989) and $50 \%$ and $33 \%$ homology, respectively, for myogenin (Wright et al. 1989) in these same regions. Human Myf5 and rat myogenin are myogenic factors related to, but distinct from, MyoD. If CMDl is not the homolog to MyoDl in chicken, it certainly is related more to MyoD1 than to either Myf5 or myogenin. The argument is even stronger if one considers the likely divergence of CMD1 and MyoD1 between birds and mammals. Alternatively, CMD1 represents a different but related myogenic factor unique to chicken skeletal muscle.

\section{CMD converts $10 T^{1} / 2$ fibroblasts into stable myoblasts}

Constructs containing either the RSV or SV40 promoter regulating CMDl expression (see Materials and methods/ were cotransfected with RSVneo as the selectable marker into $10 \mathrm{~T}^{1 / 2}$ cells. Surprisingly, no stable myoblast colonies were established with the RSV promoter regulating CMD1 expression, whereas $\sim 20-50 \%$ of the transformants expressing CMD1 under the control of the SV40 promoter formed stable myoblast colonies that fused to form multinucleated syncytia expressing skeletal muscle myosin, as judged by immunostaining with antimyosin monoclonal antibody (Fig. 3). Similar analysis of the RSV-CMD1 transformants revealed the presence of very few mononucleated, large flat cells expressing myosin (Fig. 3). These myosin-positive single cells represented $\sim 0.03-0.05 \%$ of the cell population ( $3-5$ cells per 10,000 cells counted) in both stable and transient transfections (after 4 days) and were

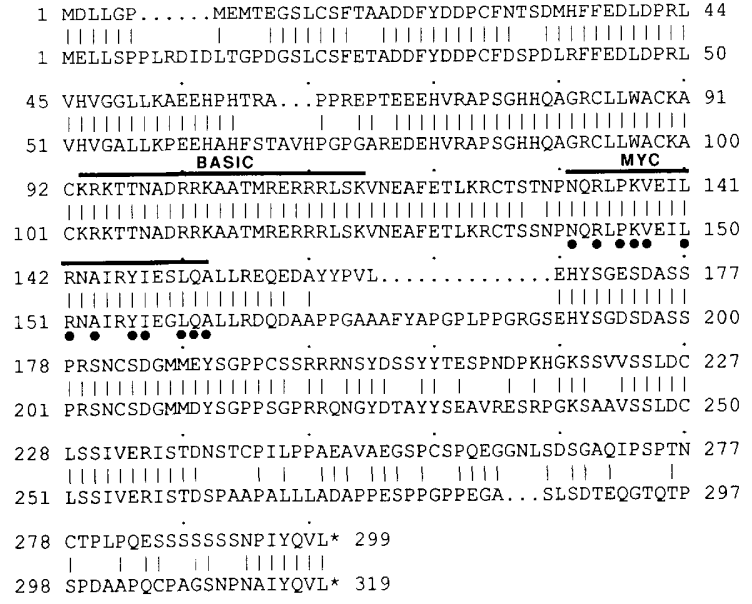

Figure 2. Amino acid sequence comparison of the avian CMD1 (top line) and the mouse MyoDl (bottom line) polypeptides. The amino acid positions of mouse MyoDl (bottom line) are used as the position reference. There is a $80 \%$ concordance in amino acid sequence with five major regions of sequence similarity; an acidic amino terminus up to amino acid 61 (acidic residues overlined); a histidine-cysteine cluster between amino acids 62 and 101; a basic region between amino acids 102 and 124; a myc homology region between amino acids 141 and 162 ; and a minor serine rich region between amino acids 190 and 224. CMDl is 298 amino acids long compared to 318 amino acids for MyoDl. The dots in the myc region mark amino acid identities with v-myc (Davis et al 1987).

seen always as isolated single cells. Wright et al. (1989) report similar frequencies for transient transfections of $10 \mathrm{~T}^{1 / 2}$ cells with myogenin under the control of the murine leukemia virus (MLV) long terminal repeat (LTR). The frequency of these positive cells with RSV-CMDl is several orders of magnitude greater than the frequency of microcolony formation, a measure of the spontaneous myogenic conversion in 10T $1 / 2$ cells (Lassar et al. 1986), and the cells were never seen in the absence of CMD1 expression. Presumably, the overexpression of the CMDl gene product induced rapid myogenic conversion in single cells; therefore, no cell replication occurred and no myoblast line was established. Three different attempts with varying ratios of RSV-CMD1 to RSVneo did not produce any stable myoblast clones with the RSV construct. In contrast, all independent transfections with the SV40-CMD1 construct produced myoblast colonies with frequencies of $\sim 20-50 \%$ conversion of the total cells.

It is of interest to note the variation in the phenotype of the myoblast colonies. Many of the colonies differentiated without the use of low calf serum or horse serum and displayed a diverse morphology, from narrow elongated myotubes to 'myobags' containing many nuclei. In every instance, the multinucleated cells were myosin positive. As reported previously for MyoDl expressed from the MLV LTR (Tapscott et al. 1988), CMD1 expressed from the SV40 promoter also activated the endogenous MyoD1 gene in 10T $1 / 2$ cells (Fig. 4). 


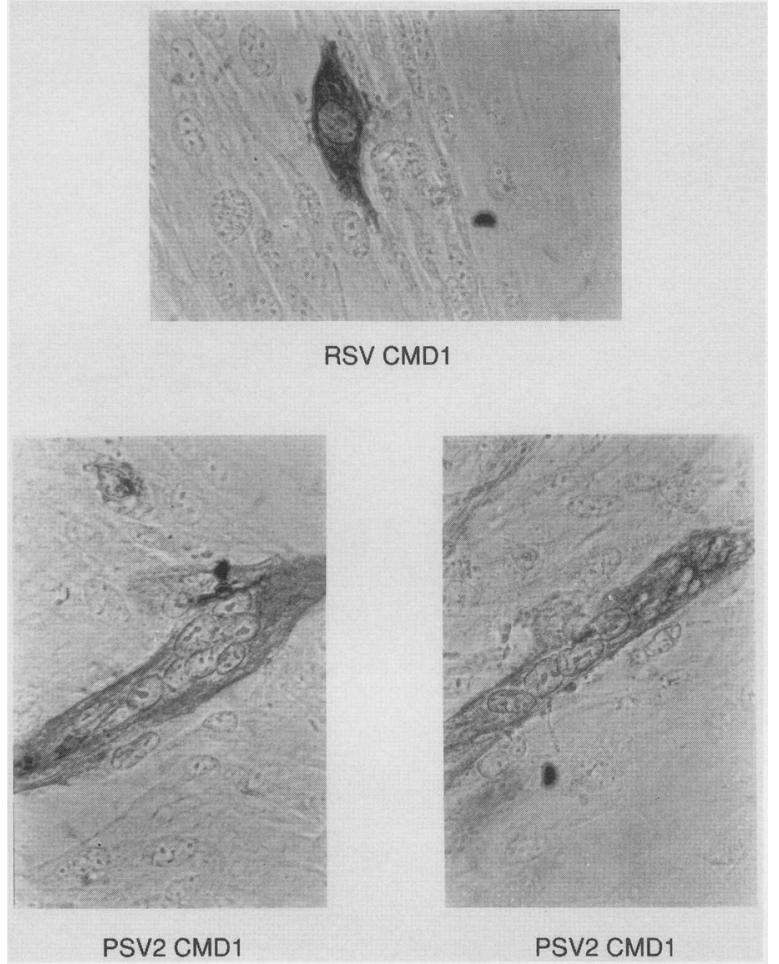

Figure 3. Anti-myosin staining of $10 \mathrm{~T}^{1 / 2}$ cells converted to myogenic cell with the expression of CMD1 under the control of the RSV promoter (RSV-CMD1) or the SV40 promoter (PSV2-CMD1). Cells treated with either RSVneo or PSV2gpt were never myosin positive or converted to myogenic cells.

Transient expression of CMD1 in nonmuscle cells can activate cotransfected muscle-specific promoters

We have shown previously that $10 \mathrm{~T}^{1 / 2}$ cells transformed stably with the chicken cardiac actin gene do not express cardiac actin mRNA transcripts, whereas transformed azacytidine-derived myoblasts express and regulate the exogenous cardiac actin gene (Lassar et al. 1986). Here, we used a cotransfection assay to determine whether CMD1 supplied in trans will activate the cardiac actin promoter joined to chloramphenicol acetyltransferase gene (CAT) (Quitschke et al. 1987, 1989a) in $10 \mathrm{~T}^{1 / 2}$ cells. When the CAT construct was transfected alone or in combination with RSVneo into $10 \mathrm{~T}^{1} / 2$ fibroblasts, no CAT activity was observed. However, cotransfection with RSV-CMD1 plasmid instead of RSVneo produced a substantial increase in CAT activity (Fig. 5A). Similar activation was seen with PSV-CMD, but the level of activation was not so great; therefore, all experiments were performed with RSV-CMD. CAT constructs with the chicken skeletal actin promoter and the myosin light-chain 3 promoter were activated also; however, these promoters normally are less active than the cardiac actin promoter in $10 \mathrm{~T}^{1 / 2}$ cells (Fig. 5A). In contrast, coactivation of so-called housekeeping or constitutive promoters regulating CAT expression, such as the $\beta$-actin promoter or the RSV LTR and the SV40 early promoter, were all affected differently in $10 \mathrm{~T}^{1 / 2}$ cells in response to CMDl expression, showing either moderate or no activation (Fig. 5A). The transcriptional start sites for the $\alpha$ - and $\beta$-actin CAT constructs in 10T $1 / 2$ cells treated with RSV-CMD1 were correct, as judged by primer extension assay (Fig. $5 \mathrm{~B}$ ). Therefore the activation of the $\beta$-actin promoter by CMDl cannot be due to the use of a different transcriptional start site. $\beta$-CAT activation by CMD1 has been seen only in $10 \mathrm{~T}^{1 / 2}$ cells and does not occur in any of the cell backgrounds described later. The transcriptional starts were not checked with the $\alpha$-skeletal and light-chain 3 promoter constructs due to the difficulty in isolating enough CAT mRNA from the transiently expressing cells. Nevertheless, $\alpha$-cardiac actin CAT transcription was initiated specifically and correctly in response to CMD1 expression in $10 \mathrm{~T}^{1} / 2$ cells.

The degree of response to CMD1 expression from

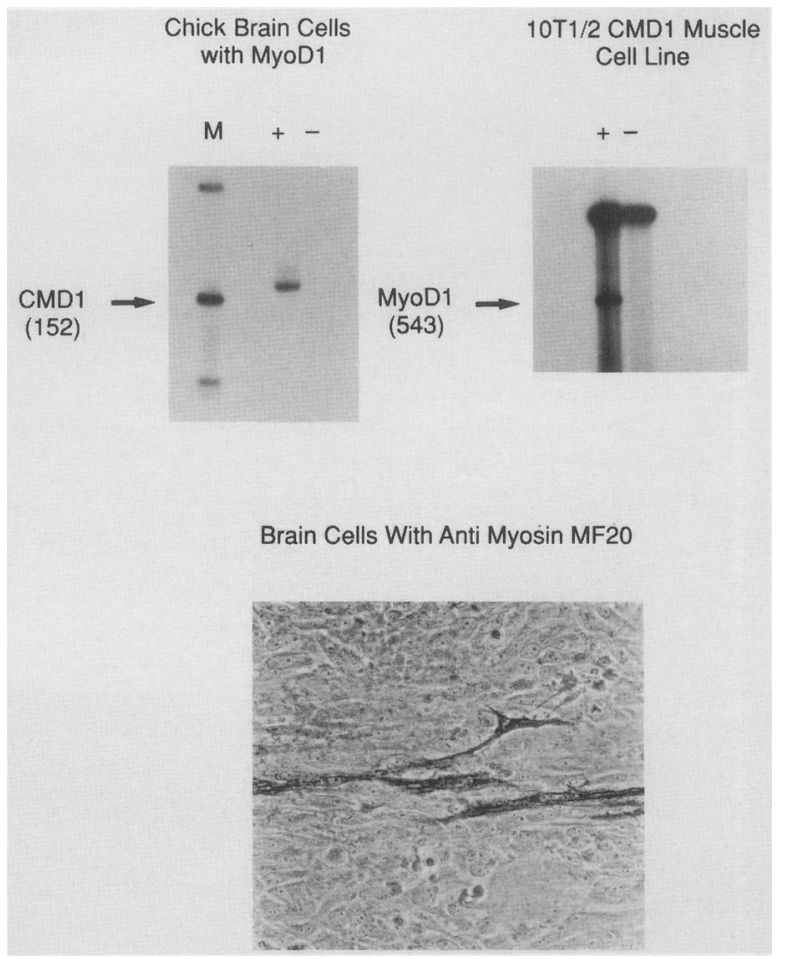

Figure 4. $S 1$ nuclease protection assay showing the endogenous activation of CMD1 and MyoDl in brain and 10T $1 / 2$ cells, respectively. (Top, left) Chick brain cells treated with RSVMyoDl (+) or RSVneo (-). (Lane $M$ ) Marker lane with three labeled fragments of MspI PBR322. The expected protected band of 152 nucleotides is seen for the expression of CMD1 RNA activated by RSV-MyoD1. (Top, right) 10T1/2 cells treated with PSV2-CMD1 $(+)$ or PSV2gpt $(-)$. The expected protected band of 543 nucleotides is seen for the expression of MyoDI RNA activated by PSV2-CMD1. The upper band in the plus $(+)$ and minus (-) lanes is reannealed probe. (Bottom) Chick brain cells treated with RSV-CMDI and stained with monoclonal antibody MF20 for skeletal muscle myosin. No myosinpositive brain cells were seen when transfected with either RSVneo or PSV2gpt. 

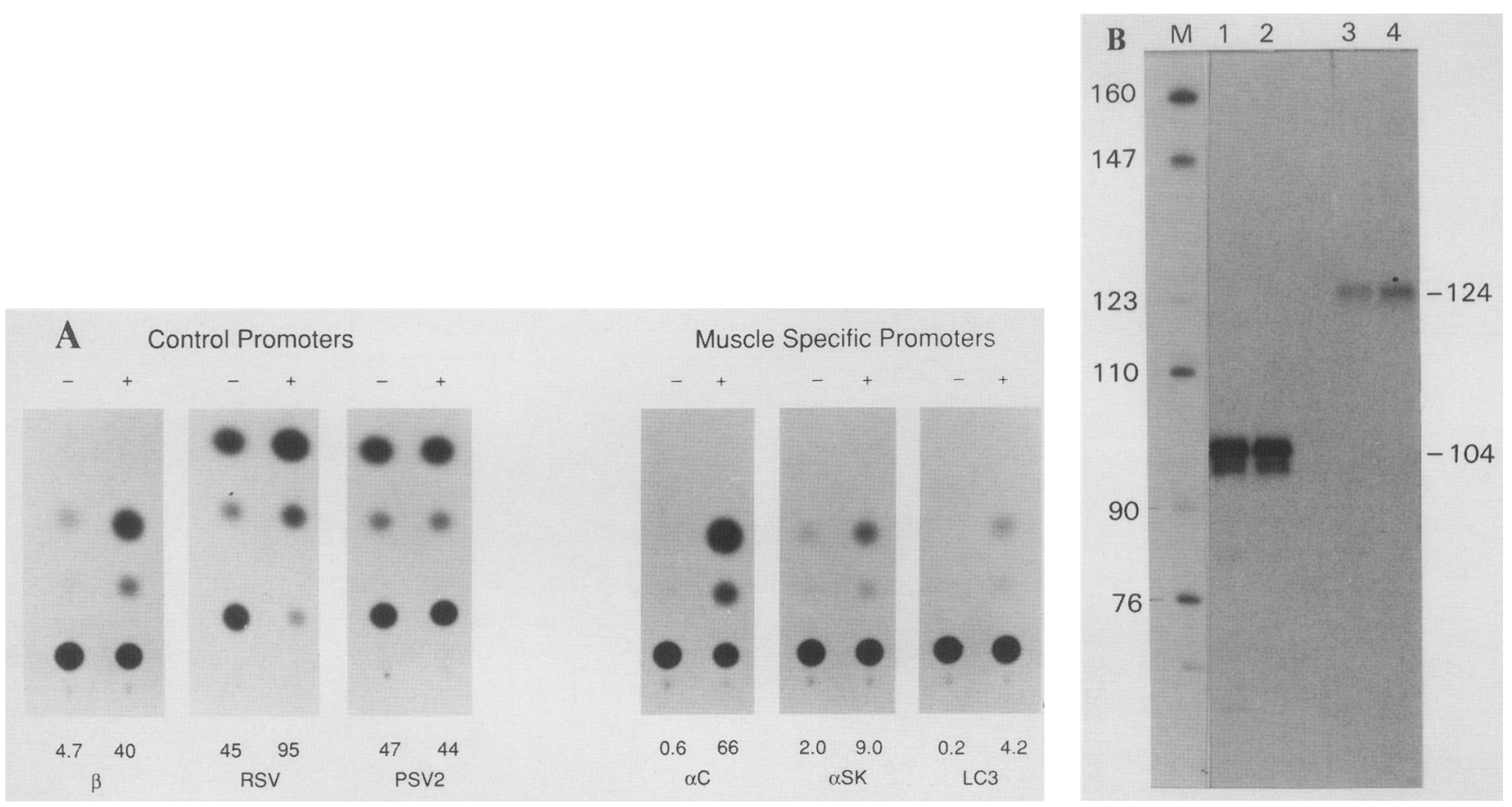

Figure 5. (A) Muscle-specific promoter activation by transient expression of cotransfected RSV-CMDI in $10 \mathrm{~T}^{1 / 2}$ cells. Control promoter CAT constructs use the chicken $\beta$-actin promoter ( $\beta$ ) (DePonti-Zilli et al. 1988), the RSV promoter (RSV), and the SV40 promoter (PSV2). The muscle-specific promoters are the chicken $\alpha$-cardiac actin promoter $(\alpha \mathrm{C})$ (Eldridge et al. 1985), the chicken $\alpha$-skeletal actin promoter ( $\alpha$ Sk) (Seiler-Tuyns et al. 1984), and the myosin light-chain 3f promoter (LC3) (Billeter et al 1988). RSVneo( - ) was used as a negative control for RSV-CMDI + ) in the cotransfections. Reaction conditions were as described previously (Billeter et al. 1988). The approximate percent conversion of $\left[{ }^{14} \mathrm{C}\right] \mathrm{chloramphenicol}$ to the acetylated form is indicated below each reaction. $(B)$ Primer extension analysis of transiently expressed CAT mRNA from the $\alpha$-cardiac and $\beta$-cytoplasmic actin CAT constructs in $10 \mathrm{~T}^{1 / 2}$ cells and in BrdU-treated cultures of embryonic chick breast muscle treated with RSV-CMD. (Lane $M$ ) MspI digest of pBR322 as marker. Primer extension of $\beta$-actin CAT mRNA from 10T $1 / 2$ cells (lane 1) and BrdU-treated myoblasts (lane 2), showing the expected 104-nucleotide product for the correct transcription initiation from the $\beta$-actin gene promoter (Quitschke et al. $1989 \mathrm{~b}$ ). Primer extension of $\alpha$-cardiac actin CAT mRNA from 10T $1 / 2$ cells, (lane 3) and BrdU-treated myoblasts (lane 4), showing the expected 124-nucleotide product for the correct transcription initiation from the $\alpha$-cardiac actin gene promoter (Quitschke et al. 1989a).

these three control promoters was also dependent on cell background (see below). The activation ratio of the cardiac actin promoter was $\sim 30$ times greater with RSV-CMD1 than with PSV2-CMD1 in the cotransfection assay (data not shown), indicating that increased levels of CMD1 in 10T $1 / 2$ cells appear to influence the level of expression from target promoters and thus may influence the rate of myoblast conversion. Levels of CMD mRNA were also substantially higher with the RSV promoter than with the SV40 promoter, as judged by the S1 assay (data not shown). Similar differences were reported for CAT mRNA expression (Gorman 1985) with the RSV and SV40 promoters.

The results of the cotransfection assay led us to consider whether muscle promoter activation was restricted to cells of mesodermal origin with a 'predetermined' potential to undergo myogenic conversion, as in the case of $10 \mathrm{~T}^{1 / 2}$ cells and its related phenotypes, or whether activation could be extended to differentiated nonmuscle cells and cells of different germ-layer origin. For these studies we chose primary embryonic chick brain cells of ectodermal origin, the HepG2 human liver cell line of endodermal origin that expresses numerous liver markers (Aden et al. 1979), CV1 kidney cells of mesodermal origin, and P19 embryonal carcinoma cells (McBurney and Rogers 1982) of pre-germ-layer origin. P19 cells give rise to cell types representative of all germ-layers in response to retinoic acid. The cardiac actin promoter CAT construct was used to measure activation in response to either RSV-CMD1 or RSVneo, and the $\beta$-actin promoter CAT construct was used as a nonresponsive constitutive control promoter. In the cell backgrounds mentioned above, the $\beta$-actin promoter does not activate in response to CMD1 expression as seen in $10 \mathrm{~T}^{1 / 2}$ fibroblasts. The activation results for brain, liver, kidney, and P19 cells are shown in Figure 6. In all instances, the cardiac actin promoter was activated in response to the transient expression of the CMDl gene product, whereas no activation above base line was seen with the $\beta$-actin promoter. In P19 cells, substantial activation of the cardiac actin promoter $130-$ fold) occurred only after 4 days in culture, and very little 
activity was observed at 2 days $(<3$-fold), the normal assay time for other cell types. Thus, activation of a muscle-specific promoter by CMD1 does not appear restricted by the embryonic germ layer or tissue-specific phenotype of the target cells. More importantly, brain cells (ectodermal in origin) were skeletal myosin positive, indicating substantial myogenic conversion in response to CMDl activation (Fig. 4). Furthermore, transfection of chick brain cells with RSV-MyoD1 (mouse) activated the endogenous CMDl (chick) gene, as judged by SI analysis (Fig. 4). The nature of the converted cell is under investigation. We analyzed only the other cell types with the coactivation assay; however, we have no evidence that would indicate incorrect transcriptional initiation is occurring on the cardiac promoter in $10 \mathrm{~T}^{1} / 2$ cells, brain cells $\langle\mathrm{S} 1$; data not shown, as these cells convert), or in BrdU-treated myoblasts (see below). The activation of the cardiac promoter in these other cell backgrounds is dependent on CMDl expression, and we have no reason to believe that transcriptional initiation should vary in these cases, given the results mentioned for $10 \mathrm{~T}^{1 / 2} \mathrm{z}$ cells, brain cells, and BrdU-treated myoblasts (see below).

\section{BrdU inhibits CMD1 and MyoD1 expression in myoblasts}

It has long been known that the thymidine analog BrdU inhibits the differentiation of a wide variety of cells (Wright 1986). Muscle is an interesting case and has probably been studied in the greatest detail (Bischoff and Holtzer 1970). Chicken myoblasts grown in BrdU do not express any differentiated markers (Paterson and Bishop 1977) and will continue to divide until the analog is removed. One cell division in the absence of BrdU is sufficient to restore the myogenic phenotype (Bischoff and Holtzer 1970). As little as a 30\% replacement of the thymidine in the genome with BrdU will block myogenesis. The kinetics of myogenic inactivation and its reversal suggested that a single locus was the target for the BrdU effect (O'Neill and Stockdale 1974). Previous work from this lab (Billeter et al 1988) has demonstrated that muscle-specific promoters will not function in myoblasts grown in BrdU whereas housekeeping or constitutive promoters such as the $\beta$-actin promoter or the RSV LTR function normally in the presence or absence of the analog. Using the cotransfection assay described above, we wanted to determine whether CMDl supplied in trans could activate a muscle-specific promoter CAT construct in primary embryonic chick breast muscle cultures and in $\mathrm{C} 2 \mathrm{C} 12$ myoblasts grown in BrdU. The $\beta$-actin and RSV promoter CAT constructs were used as constitutive nonmuscle control promoters. As shown in Figure 7, the cardiac and skeletal actin promoters and the myosin light-chain 3 promoter were activated by CMD1 supplied in trans in BrdU-treated chick myoblasts, whereas the $\beta$-actin promoter was suppressed approximately twofold. In $\mathrm{C} 2 \mathrm{C} 12$ myoblasts the cardiac actin promoter was activated also, the $\beta$-actin promoter was suppressed again, and the RSV-CAT construct was not affected by CMD1 expression. CMD1 in trans can reactivate muscle-specific promoters in BrdU-treated myoblasts; and in the case of the two strongest promoters, the $\alpha$-cardiac and $\beta$-actin CAT constructs, primer extension analysis of the transcriptional starts demonstrated correct initiation (Fig. 5B). The $\alpha$-skeletal and light-chain 3 promoters were not checked as a result of the difficulty in isolating enough CAT mRNA from the transiently expressing cells. Nevertheless, activation is specific and correct for the $\alpha$-cardiac and $\beta$-actin promoters, as seen in $10 \mathrm{~T}^{1} / 2$ cells.

These findings suggested that BrdU was blocking the expression of CMD1 and MyoD1. S1 nuclease protection analysis of BrdU-treated and control chicken and mouse myoblasts revealed that the endogenous levels of CMD1 and MyoDl were indeed substantially lower in treated myoblasts, yet levels of $\beta$-actin RNA, transcribed from a BrdU-insensitive promoter (Billeter et al. 1988), were identical in the presence or absence of the analog (Fig. 8). These results are consistent with the notion that BrdU inhibits myogenesis by blocking the expression of the

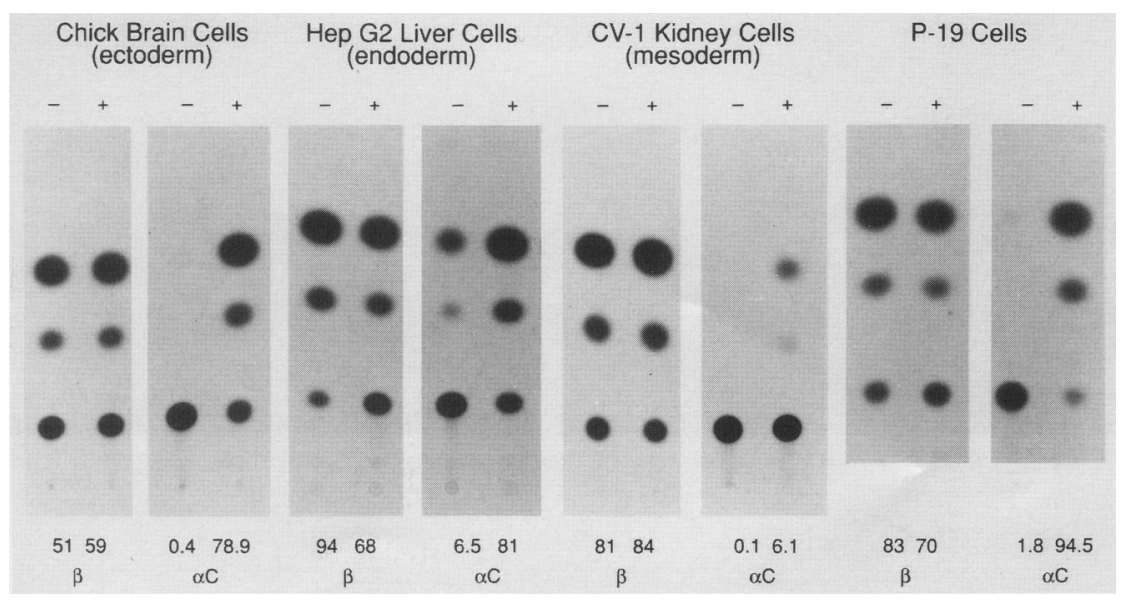

Figure 6. Muscle-specific promoter activation in nonmuscle cells from different embryonic germ-layer origins. The control promoter CAT construct contained the chicken $\beta$-actin gene promoter $(\beta)$. The $\alpha$-cardiac actin promoter CAT plasmid $(\alpha \mathrm{C})$ was used to measure activation of a muscle-specific promoter when cotransfected either with RSVneo $(-)$ or RSV-CMD1 $(+)$ in chick embryonic brain cells (ectoderm), HepG2 liver cells (endoderm), CV-1 kidney cells (mesoderm), and P19 embryonal carcinoma cells (pre-germlayer). The approximate percent conversion is indicated below each reaction, as described in the legend to Fig. 5. 


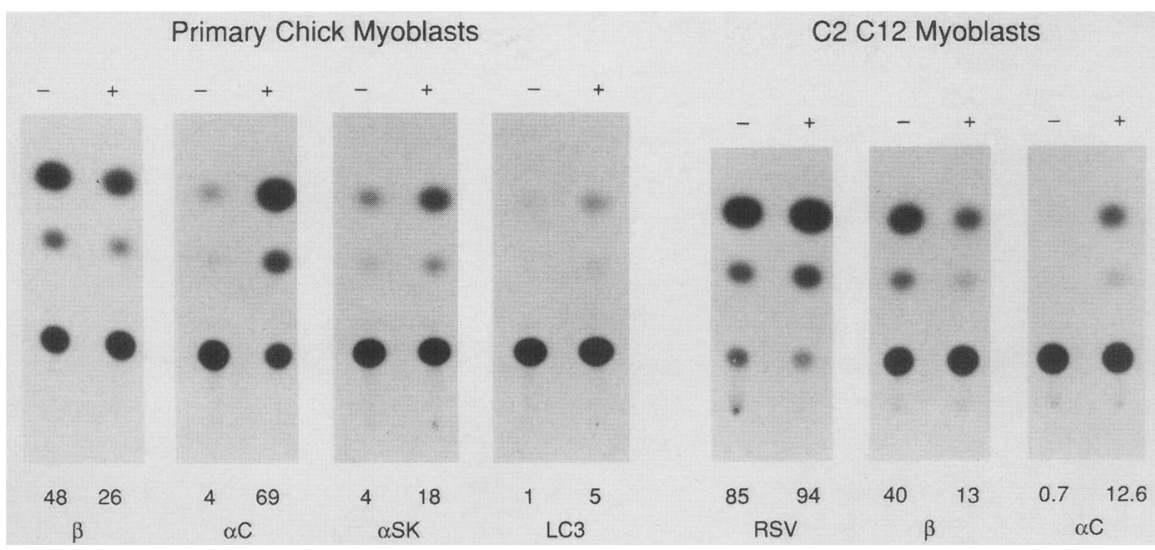

Figure 7. Muscle-specific promoter activation in BrdU-treated chicken and mouse myoblasts. Control promoter CAT constructs included the RSV promoter (RSV) and the chicken $\beta$-actin promoter ( $\beta$ ). Muscle-specific promoter CAT constructs included the chicken $\alpha$-cardiac actin promoter $(\alpha \mathrm{C})$, the chicken $\alpha$-skeletal actin promoter $(\alpha \mathrm{Sk})$, and the myosin light-chain 3f promoter (LC3), and were cotransfected with either RSVneo $(-\mid$ or RSV-CMDI $|+|$. The approximate percent conversion is indicated below each reaction.

myogenic factors $\mathrm{CMDl}$ and $\mathrm{MyoDl}$, which act directly or together with other factors to promote myogenesis.

\section{Discussion}

Although much is being learned about the molecular basis of gene regulation during differentiation, very little is known about the developmental processes that lead to tissue diversity. Nothing is known about what determines tissue-specific phenotype or how 'fixed' phenotype is once it is established. With regard to the latter issue, the work of Blau and associates (Blau et al. 1985) has shown that the differentiated state is not fixed irreversibly but can respond, to a degree, to positive transacting factors that can change the pattern of gene expression. In this instance heterokaryons formed between mouse $\mathrm{C} 2 \mathrm{C} 12$ myotubes and a variety of human cell types demonstrated the activation of human musclespecific genes in the absence of DNA replication in all cells tested, and this was independent of the germ-layer origin or the degree of differentiation of the human cells. However, factors involved in the activation have not been identified so far. The isolation and characterization of the myogenic factor MyoD1 (Davis et al. 1987), myogenin (Wright et al. 1989), and myf5 (Braun et al. 1989), single gene products capable of activating the entire muscle tissue phenotype in mouse $10 \mathrm{~T}^{1 / 2}$ fibroblasts, may be the first examples of such factors. Our results and those mentioned clearly demonstrate that various myogenic factors similar to MyoDl are found in the vertebrates from chicken to man and myogenic conversion likely involves more than a single gene product.

We isolated a single cDNA clone, CMDl, from an embryonic avian skeletal muscle cDNA library in $\lambda g t 10$ that is structurally very similar to and functionally identical with mouse MyoDl. The amino acid sequences of the two polypeptides are $80 \%$ concordant and cover five major domains of sequence homology, including one of $>90$ amino acids with $>95 \%$ homology, which contains the basic and myc domains essential for myoblast conversion of $10 \mathrm{~T}^{1} 1 / 2$ fibroblasts (Tapscott et al. 1988). CMD1, which is 20 amino acids shorter than MyoD1, converted $10 \mathrm{~T}^{1} / 2$ fibroblasts to stable myoblasts with an efficiency similar to MyoD1 $(\sim 20-50 \%)$ in cotransfections, as long as the level of CMD1 expression was regulated appropriately with the use of the correct viral promoter. This was inferred from the observation that under the control of the strong RSV promoter, CMDl never gave stable myoblast colonies, whereas use of the early SV40 promoter, a weaker promoter, gave conversion results similar to MyoDl with the MLV LTR. The SV40 construct was also less effective in the activation assay (30-fold less), suggesting that levels of $\mathrm{CMDl}$ are important in controlling the rate of myoblast conversion by regulating muscle-specific genes. Lassar and associates (Davis et al. 1987) reported that MyoDl appears to antagonize cell growth because the number of stable transformants with MyoDl was $\sim 10$-fold lower than cells transfected with the Neo selection vector alone: In this instance transformation efficiency could be increased if fibroblast growth factor (FGF), an antagonist to withdrawal from the cell cycle and commitment to myogenesis, was included in the transformation protocol (Davis et al. 1987). These findings suggest that $\mathrm{CDMl}$ and related factors may modulate entry into the cell cycle because overexpression of CMDl under the RSV promoter, unlike the SV40 promoter, resulted in the rapid single-cell commitment of the $10 \mathrm{~T}^{1 / 2} / 2$ fibroblast to myogenesis. The presence of myosin-positive single cells seen with the RSV-CMD1 transfections and the lack of stable myogenic colonies would support this interpretation.

$\mathrm{CH} 310 \mathrm{~T}^{1 / 2}$ fibroblasts are mesodermal in origin and give rise to characteristic mesodermal cell types when treated with 5-azacytidine. The action of CMD1 or MyoD1 could have been restricted to cells of mesodermal origin. Myogenic conversion of $10 \mathrm{~T}^{1 / 2} \mathrm{z}$ cells with PSV2-CMD1 indicates clearly that there is no species 


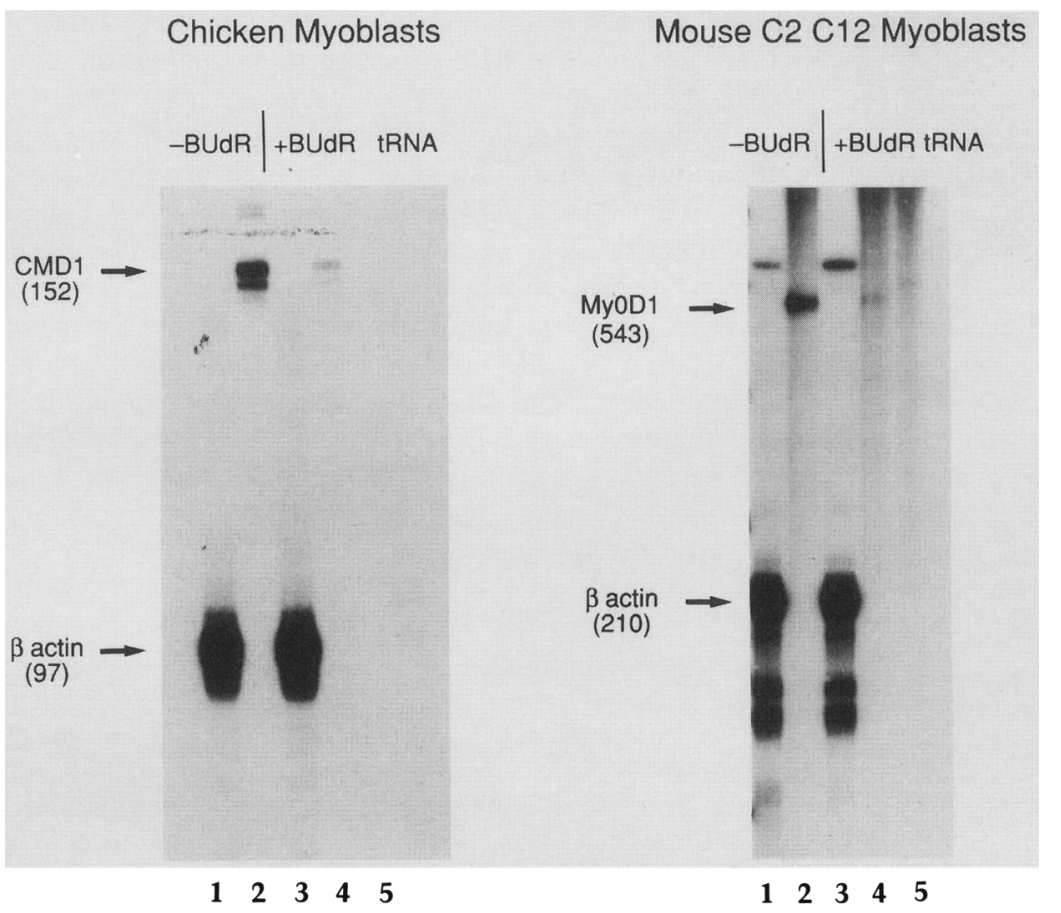

Figure 8. S1 nuclease protection assay for the BrdU inhibition of CMDl and MyoDl expression in myoblasts. Ten micrograms of total RNA was prepared and assayed, as described (DePontiZilli et al. 1988). The protected fragments (lanes 2 and 4) for CMD1 and MyoD1 are 152 and 543 bp, respectively. The $\beta$-actin probes protect fragments (lanes 1 and 3) of 97 and $210 \mathrm{bp}$ for the chicken and mouse $\beta$-actin RNAs, respectively. The probe for chicken $\beta$-actin is specific for the $5^{\prime}$ UTR, whereas the mouse $\beta$-actin probe is labeled at a BgIII site in the coding portion of the cDNA (Alonso et al. 1986) and will cross-react with other actin transcripts (additional bands seen in $\beta$-actin in $\mathrm{C} 2 \mathrm{C} 12$ cells). The last lane (lane 5) is a tRNA control. barrier to the action of CMD1. Our preliminary results on muscle-specific promoter activation and skeletal muscle myosin synthesis in differentiated cells of nonmesodermal origin suggest that the action of CMD1 and related myogenic factors is not restricted by the germlayer origin of the target cell. It is difficult to know whether the transient activation of muscle-specific promoter-dependent CAT activity per se is a measure of myogenic conversion. The strong, correct activation of the cardiac actin promoter expressing CAT and the concomitant conversion of $10 \mathrm{~T}^{1 / 2}$ fibroblasts to stable myoblasts suggests that muscle-specific promoter-dependent CAT activity may be a measure of myogenic conversion. Similar activation of the cardiac actin promoter-dependent CAT activity, the endogenous skeletal muscle myosin synthesis, and the endogenous activation of CMD1 in brain cells strengthens this correlation. This is reflected also in the greater level of muscle promoter-dependent CAT activity, the faster rate of myoblast conversion, and the myogenic commitment of $10 \mathrm{~T}^{1 / 2}$ cells with the RSV versus the SV40 promoter regulating the expression levels of CMD1. As a first approximation, muscle-specific promoter CAT activation would seem to be a good indicator of the potential myogenic conversion of a particular cell type.

Because CAT activity is a more sensitive assay for putative conversion than antimyosin staining, this may account for the previous failure to detect any measure of myogenic transformation in CV1 kidney cells (Davis et al. 1987). We were unable to detect any antimyosin activity in CVl cells. In our hands, CV1 cells gave very low-level activation of the cardiac actin promoter in response to RSV-CMD1; therefore, it is unlikely that this would appear as antimyosin staining if the penetrance of the conversion was not sufficient. The activation of endogenous muscle-specific genes is the most dramatic evidence of myogenic conversion; this is being investigated further in HepG2 liver cells and in other cell types. At present, because we have no evidence for the expression of endogenous muscle genes (antimyosin staining) in HepG2 liver cells or in P19 cells, activation may be distinct from conversion, the latter being more restricted in a particular cell background. Alternatively, this lack of antimyosin staining may reflect the rate of myoblast conversion, and this may depend on the cell phenotype. The timing of cardiac actin promoter activation in P19 cells suggests that cells can respond to CMD1 at different rates. The heterokaryon studies by Blau (Blau et al. 1985) indicated that the germ-layer origin of the human cells had a marked effect on the rate and level of expression of human muscle genes, as judged by the expression of muscle surface antigen, $5.1 \mathrm{Hll}$. There was a decreased response going from fibroblasts (mesoderm) to keratinocytes (ectoderm) and from keratinocytes to hepatocytes (endoderm). If this is the case, a more sensitive assay other than the CAT assay, such as a direct measure of muscle-specific transcripts, might reveal the endogenous activation of muscle genes in HepG2 cells and in P19 cells.

The experiments presented with the BrdU-treated myoblasts provide additional evidence that the BrdU inhibition of myogenesis likely acts by reducing the expression levels of CMDl and MyoDl in chicken and mouse myoblasts, respectively. In support of this hypothesis, Davis et al. (1987) reported that there is a significant reduction in the level of MyoDl expression in all nondifferentiating azacytidine-derived myoblast variants examined and concluded that a minimal level 
of MyoDl was required to maintain the myoblast phenotype. The fact that CMD1 expression in BrdU-treated myoblasts can restore muscle-specific promoter activity is consistent with this interpretation. Our BrdU experiments may explain the results of Wright and Aronoff (1983), which demonstrated the induction of rat myosin light-chain $\mathbf{l}$ in BrdU-grown rat myoblasts fused to chicken binucleated myotubes.

It is not clear whether CMD1 or MyoDl alone is responsible for myogenic determination in these cells. The results of Pinney et al. (1988), Wright et al. (1989), and Braun et al. (1989) and the preliminary identification of the myogenic determination genes Myd1, myogenin, and Myf5 suggest that conversion involves the interplay of multiple genes. Because CMD1, Myd1, Myf5, and MyoDl can activate MyoD1 in 10T $1 / 2$ cells, and MyoD1 can activate CMD1 in chick cells, interdependent regulatory pathways involving different myogenic factors appear to be operative. It is certainly interesting to speculate that the inhibitory effect of BrdU on the differentiated functions of a variety of cell types may involve very few genes similar in nature to CMD1 and MyoD1.

The mechanism of action of CMDl and MyoDl has not been determined yet. Early efforts to measure possible binding of CMD1 synthesized in vitro in a reticulocyte lysate from SP6 RNA transcripts to various musclespecific promoters have not been successful in our studies. This may not be surprising because MyoDl appears to be a phosphorylated protein and may require precise phosphorylation for its function (Tapscott et al. 1988). This would not occur during cell-free synthesis. Alternatively, the reticulocyte lysate product may not be active. We have not been able to synthesize active AceI, the copper-dependent transcriptional activator of the yeast cup1 gene, in reticulocyte lysates (Fürst et al. 1988), even though synthesis in wheat-germ extracts gives an active product. Previous studies (Vrana et al. 1988) on TFIIIA alude to a similar result with reticulocyte versus wheat-germ lysates. Several lines of evidence suggest that these myogenic factors are not involved directly in muscle-specific promoter function. The cardiac actin gene is expressed both in the heart and in early developing skeletal muscle (Minty et al. 1982; Paterson and Eldridge 1984); however, there is no detectable CMDl or MyoDl expression in cardiac muscle (Davis et al. 1987, and this paper/. Transient expression of CMD1 during development cannot be ruled out as a mechanism of cardiac actin gene activation in the heart, but CMD1 is not required for continued expression of cardiac-specific genes. The varying activity of housekeeping or constitutive promoter CAT constructs in response to CMD1 expression in different cell backgrounds suggests that CMDl can modulate factors regulating these promoters and may not act directly on DNA. The variation that is seen in the response of the $\beta$-actin promoter does not reflect a change in the transcriptional start (Fig. 5B). CMD1 is expressed in myoblasts, yet transfected (Quitschke et al. 1987, 1989a), and endogenous muscle genes (Lassar et al. 1986) are repressed prior to cell-cycle withdrawal and fusion; therefore, the presence of CMD1 per se does not trigger promoter activation. In this instance, the levels of CMD1, as suggested here, may play a crucial role in regulating myogenesis and, indirectly, the activity of muscle genes. Secondary modifications of CMD1 may also modulate it effects on myogenesis. This will have to be determined. Recent results of A.B. Lassar and associates (pers. comm.) demonstrate that because a fusion protein with the MyoD domain can bind to the muscle creatine phosphokinase enhancer (Sternberg et al. 1988) in a specific manner, direct DNA binding may be involved in some instances in MyoD and CMD1 myogenic activation.

\section{Materials and methods}

Isolation and sequencing of CMD1

A cDNA library was prepared from fused cytosine arabinoside (araC)-treated cultures of embryonic chick breast muscle in $\lambda \mathrm{gt10}$, as described previously (Huynh et al. 1985). Approximately $2 \times 10^{6}$ plaques were screened in duplicate with the ${ }^{32} \mathrm{P}$ nick-translated 1.8-kb mouse MyoDl insert (Davis et al. 1987), prepared using standard procedures (Maniatis et al. 1982). Filters were hybridized in $5 \times$ SSC, $5 \times$ Denhardt's solution, 50 $\mathrm{mM}$ sodium phosphate buffer $(\mathrm{pH} 7.0), 43 \%$ deionized formamide, $1.0 \%$ SDS, $250 \mu \mathrm{g} / \mathrm{ml}$ denatured calf thymus DNA with $10^{7} \mathrm{cpm}$ of probe per ten $150 \mathrm{~mm}$ nitrocellulose filters. Filters were hybridized for 2 days at $37^{\circ} \mathrm{C}$, washed twice at room temperature with $2 \times \mathrm{SSC}, 0.1 \%$ SDS for $15 \mathrm{~min}$, and twice at $50^{\circ} \mathrm{C}$ in the same wash buffer for $30 \mathrm{~min}$ per wash (McGinnis et al. 1984). Phage were plaque-purified, and the $1.5-\mathrm{kb}$ EcoRI insert of CMD1 was subcloned into Bluescript KS (Stratagene) in both orientations for restriction analysis and sequencing. The nucleotide sequence was determined using the procedures of Sanger et al. (1977), with the direct and reverse primers in the KS plasmid and the T7 DNA polymerase system (Pharmacia). Sequence was analyzed and assembled with the Staden programs (Staden 1984) and the GCG Sequence Analysis Software Package (University of Wisconsin) (Devereux et al. 1984).

\section{Expression of CMD1 in eukaryotic cells}

The 1.5-kb EcoRI insert from CMD1 or the 1.8-kb insert from MyoDl (Davis et al. 1987) was subcloned in Bluescript KS and reisolated as a HindIII $\left(5^{\prime}\right)-S m a I\left(3^{\prime}\right)$ fragment for further subcloning into either RSVneo or PSV2gpt cut with HindIII-SmaI to remove the selectable marker genes (Gorman 1985). These constructs provided the appropriate promoter elements and processing signals for CMDl and MyoDl expression in eukaryotic cells.

\section{Cells and conditions for DNA transfection}

$\mathrm{CH} 3$ 10T $1 / 2$ mouse fibroblasts, HepG2 human liver cells, and CV-1 monkey kidney cells were obtained from the American Type Culture Collection. P19 embryonal carcinoma cells were a generous gift of M. McBurney (McBurney and Rogers 1982). Chick primary cultures of 12-day embryonic breast and brain were prepared as described previously (Paterson and Strohman 1972). All mammalian cells were grown in Dulbecco's modified Eagle's medium (DMEM) with $10 \%$ fetal calf serum. Primary chick cells were grown in DMEM supplemented with $10 \%$ horse serum and $2 \%$ embryo extract, as described (Paterson and Strohman 1972). Differentiation in $10 \mathrm{~T}^{1 / 2}$ cells was induced by 
incubation in DMEM with $2-10 \%$ horse serum. Stable transformants were generated by cotransfecting $10 \mathrm{~T}^{1 / 2}$ cells with $0.5-1.0 \mu \mathrm{g}$ of supercoiled RSVneo plasmid and $20-30 \mu \mathrm{g}$ of supercoiled RSV or PSV2-CMD1. Forty-eight hours after transfection, cells were grown in $0.4 \mu \mathrm{g} / \mathrm{ml}$ of G418 until selection was complete. Reactions were carried out with $\sim 10^{6}$ cells in a T-75 flask fed for 3-6 hr prior to transfection. Calcium phosphate precipitates of DNA were prepared as described /Gorman 1985); however, the DNA was allowed to stay on the cells up to $24 \mathrm{hr}$ and the glycerol shock was omitted. G418-selected cells were plated at clonal density, and the media was changed to differentiation media as soon as microcolonies were visible. Cells were washed with phosphate-buffered saline and fixed for antimyosin staining, as described (Davis et al. 1987). Antimyosin monoclonal antibody was a generous gift from $\mathrm{A}$. Lassar (MF20 from D. Fischman) and H. Blau (4a-1025). Cells were stained with second antibodies tagged with peroxidase and scored at 50 -fold magnification. For the transient coactivation assay with muscle-specific and control promoters, transfections were carried out and CAT activity was determined according to Gorman (1985), except that the glycerol shock was limited to 2 min for chick cells and was not used in mammalian cell transfections. All CAT assays were normalized to equivalent levels of luciferase activity from an internal control plasmid, PSV232AL-A $\Delta 5$, containing the SV40 enhancerless promoter driving the luciferase gene (de Wet et al. 1987). Luciferase assays were carried out on a Monolite 2001, as described (de Wet et al. 1987). Cell extracts were prepared 2-4 days after transfection. Where indicated, cells were grown in $5 \mu \mathrm{g} / \mathrm{ml}$ BrdU as described previously (Billeter et al. 1988).

\section{Nuclease S1 protection and primer extension assays}

Ten micrograms of total RNA was prepared and hybridized as described (De Ponti-Zilli et al. 1988), with 10,000-20,000 cpm of end-labeled probe prepared with $\mathrm{T} 4$ polynucleotide kinase according to standard protocol (Maniatis et al. 1982). CMD1 and $\mathrm{MyoDl}$ probes were end-labeled at convenient $\mathrm{NcoI}$ and StuI sites, respectively, to produce protected probes of $152 \mathrm{bp}$ for CMDl and $543 \mathrm{bp}$ for MyoDl. $\beta$-Actin was used as the internal standard for the $S 1$ analyses. Chicken and mouse S1 probes were prepared as described (De Ponti-Zilli et al. 1988). Primer extensions were carried out as described (Cao et al. 1987), using MMLV reverse transcriptase (BRL) with 20-40 $\mu \mathrm{g}$ of total RNA and a kinased CAT primer (Maniatis et al. 1982) from position 4921-4944 in PSV2CAT (Gorman 1985). The $\alpha$ cardiac actin and $\beta$-actin CAT promoter constructs are as described elsewhere (Quitschke et al. 1989a,b).

\section{Acknowledgments}

We thank A. Lassar and $\mathrm{H}$. Weintraub for the mouse MyoDl cDNA clone. We thank M. McBurney for P19 cells, and H. Blau and D. Fischman for antimyosin antibodies. This generosity is appreciated greatly.

\section{References}

Aden, D.P., A. Fogel, S. Plotkin, I. Damjanor, and B.S. Knowles. 1979. Controlled synthesis of $\mathrm{HBsA}_{9}$ in a differentiated human liver carcinoma-derived cell line. Nature 282: 615620.

Alonso, S., A. Minty, Y. Bourlet, and M. Buckingham. 1986. Comparison of three actin coding sequences in the mouse; evolutionary relationships between the actin genes of warm-blooded vertebrates. J. Mol. Evol, 23: 11-23.

Billeter, R., W. Quitschke, and B.M. Paterson. 1988. Approximately 1 kilobase of sequence $5^{\prime}$ to the two myosin lightchain $1_{\mathrm{f}} / 3_{\mathrm{f}}$ gene cap sights is sufficient for differentiation-dependent expression. Mol. Cell. Biol. 8: 1361-1365.

Bischoff, R. and H. Holtzer. 1970. Inhibition of myoblast fusion after one round of DNA synthesis in 5-BrdU. 1 . Cell Biol. 44: $134-150$.

Blau, H.M., G.K. Pavlath, E.C. Hardeman, C.-D. Chiu, L. Silberstein, S.G. Webster, S.C. Miller, and C. Webster. 1985 Plasticity of the differentiated state. Science 230: 758-766.

Braun, T., G. Buschhausen-Denker, E. Bober, E. Tannich, and H.H. Arnold 1989. A novel human muscle factor related to but distinct from MyoD1 induces myogenic conversion in $10 \mathrm{~T}^{1 / 2}$ fibroblasts. EMBO J. 8: $701-709$.

Cao, Z., E.A. Barron, A.J. Carrillo, and Z.D. Sharp. 1987. Reconstitution of cell-type-specific transcription of the rat prolactin gene in vitro. Mol. Cell. Biol. 7: 3402-3408.

Cavener, D.R. 1987. Comparison of the consensus sequence flanking translational start sites in Drosophila and vertebrates. Nucleic Acids Res. 15: 1353-1361.

Davis, R.L., H. Weintraub, and A.B. Lassar. 1987. Expression of a single transfected cDNA converts fibroblasts to myoblasts. Cell 51: 987-1000.

De Ponti-Zilli, L., A. Seiler-Tuyns, and B.M. Paterson. 1988. A 40-base-pair sequence in the 3 ' end of the $\beta$-actin gene regulates $\beta$-actin mRNA transcription during myogenesis. Proc. Natl. Acad. Sci. 85: 1389-1393.

Devereux, J., P. Haeberli, and O. Smithies. 1984. A comprehensive set of sequence analysis programs for the VAX. Nucleic Acids Res. 12: 387-395.

de Wet, J.R., K.V. Wood, M. Delucr, D.R. Helinski, and S. Subramani. 1987. Firefly luciferase gene: Structure and expression in mammalian cells. Mol. Cell. Biol. 7: 725-737.

Eldridge, J.D., Z. Zehner, and B.M. Paterson. 1985. Nucleotide sequence of the chicken cardiac $\alpha$-actin gene: Absence of strong homologies in the promoter and $3^{\prime}$-untranslated regions with the skeletal alphaactin sequence. Gene 36: 5563.

Fürst, P., S. Hu, R. Hackett, and D. Hamer. 1988. Copper activates metallothionein gene transcription by altering the conformation of a specific DNA binding protein. Cell 55: 705-717.

Gorman, C.M. 1985. High efficiency gene transfer into mammalian cells. In DNA cloning II (ed. D.M. Glover), pp. 143-190. IRL Press, Washington, D.C.

Hope, I.A. and K. Struhl. 1985. GCN 4 protein, synthesized in vitro, binds His3 regulatory sequences: Implications for general control of amino acids biosynthetic genes in yeast. Cell 43: $177-188$.

Huynh, T.V., R.A. Young, and R.W. Davis. 1985. DNA cloning; a practical approach. In DNA Cloning $I$ (ed. D.M. Glover), pp. 49-78, IRL Press, Washington, D.C.

Konieczny, S.F. and C.P. Emerson. C.P. 1984. 5-Azacytidine induction of stable mesodermal stem cell lineages from $10 \mathrm{~T}^{1 / 2}$ cells: Evidence for regulatory genes controlling determination. Cell 38: 791-800.

Lassar, A.B., B.M. Paterson, and H. Weintraub. 1986. Transfection of a DNA locus that mediates the conversion of $10 \mathrm{~T}^{1 / 2}$ fibroblasts to myoblasts. Cell 47: 649-656.

Maniatis, T., E.F. Fritsch, and J.C. Sambrook. 1982. Molecular cloning: A laboratory manual. Cold Spring Harbor Laboratory, Cold Spring Harbor, New York.

McBurney, M.W. and B.J. Rogers. 1982. Isolation of male em- 
Lin et al.

bryonal carcinoma cells and their chromosome replication patterns. Dev. Biol. 89: 503-508.

McGinnis, W., M.S. Levine, E. Hafen, A. Kuroiwa, and W.J. Gehring. 1984. A conserved DNA sequence in homoerotic genes of the Drosophila antennapedia and bithorax complexes. Nature 308: 428-433.

Minty, A.J., S. Alonso, M. Caravatti, and M.E. Buckingham. 1982. A fetal skeletal muscle actin mRNA in the mouse and its identity with cardiac actin mRNA. Cell 30: 185-191.

O'Neill, M.C. and F.E. Stockdale. 1974. 5-BrdU inhibition of differentiation. Kinetics of inhibition and reversal in myoblasts. Dev. Biol. 37: 117-132.

Paterson, B.M. and R.C. Strohman. 1972. Myosin synthesis in cultures of differentiating chicken embryo skeletal muscle. Dev. Biol. 29: 113-138.

Paterson, B.M. and J.O. Bishop. 1977. Changes in the mRNA population of chick myoblasts during myogenesis in vitro. Cell 12: 751-765.

Paterson, B.M. and J.D. Eldridge. 1984. $\alpha$-Cardiac actin is the major sarcomeric isoform expressed in embryonic avian skeletal muscle. Science 224: 1436-1438.

Pinney, D.F., S.M. Pearson-White, S.F. Konieczny, K.E. Latham, and C.P. Emerson. 1988. Myogenic lineage determination and differentiation: Evidence for a regulatory gene pathway. 1988. Cell 53: 781-793.

Quitschke, W.Q., J.D. Eldridge, and B.M. Paterson. 1987. Tissue specific expression of sarcomeric and cytoplasmic actin does not depend on a particular CAAT sequence element. In Mechanisms of control of gene expression, UCLA Symp. Mol. Cell. Biol. (ed. B. Culler, L.P. Goje, M.A.Q. Siddiqui, A.M. Skolka and H. Weissbach), vol. 67. Alan R. Liss, New York.

Quitschke, W.Q., L. DePonti-Zilli, Z.Y. Lin, and B.M. Paterson. 1989a. Identification of two nuclear factor binding domains in the chicken cardiac actin promoter: Implications for the regulation of the gene. Mol. Cell. Biol. (in press).

- 1989b. The beta actin promoter: High levels of transcription depend on a CCAAT binding factor. J. Biol. Chem. (in press).

Sanger, F., S. Nicklen, and A.R. Coulson. 1977. DNA sequencing with chain terminating inhibitors. Proc. Natl. Acad. Sci. 74: 5463-5467.

Seiler-Tuyns, A., J.D. Eldridge, and B.M. Paterson. 1984. Expression and regulation of chicken actin genes introduced into mouse myogenic and nonmyogenic cells. Proc. Natl. Acad. Sci. 81: 2980-2984.

Staden, R. 1984. Automation of the computer handling of gel reading data produced by the shotgun method of DNA sequencing. Nucleic Acids Res. 10: 4731-4751.

Sternberg, E.A., G. Spizz, W.M. Perry, D. Vizard, T. Weil, and E.N. Olson. 1988. Identification of upstream and intragenic regulatory elements that confer cell-type-restricted and differentiation-specific expression on the muscle creatine $\mathrm{ki}$ nase gene. Mol. Cell. Biol. 8: 2896-2909.

Tapscott, S.J., R.L. Davis, M.J. Thayer, P.-F. Cheng, H. Weinstraub, and A.B. Lassar. 1988. MyoDl: A nuclear phosphoprotein requiring a myc homology region to convert fibroblasts to myoblasts. Science 242: 405-411.

Taylor, S.M. and P.A. Jones. 1979. Multiple new phenotypes induced in $10 \mathrm{~T}^{1 / 2}$ cells and $3 \mathrm{~T} 3$ cells treated with 5 -azacytidine. Cell 17: 771-779.

Vrana, K.E., M.E.A. Churchill, T.D. Tullius, and D.D. Brown. 1988. Mapping functional regions of transcription factor TFIIIA. Mol. Cell. Biol. 8: 1684-1696.

Wright, W.E. 1986. BrdU, probability and cell variants: towards a molecular understanding of the decision to differentiate. BioEssays 3: 245-248.

Wright, W.E. and J. Aronoff. 1983. Regulation of rat myosin light-chain synthesis in heterokaryons between 5-bromodeoxyuridine-blocked rat myoblasts and differentiated chick myocytes. J. Cell Biol. 100: 311-316.

Wright, W.W., D. Sassoon, V.K. Lin. 1989. Myogenin, a factor regulating myogenesis, has a domain homologous to myoD. Cell 56: 777-783.

Zehner, Z. and B.M. Paterson. 1983. Characterization of the chicken vimentin gene: single copy gene producing multiple mRNAs. Proc. Natl. Acad. Sci. 80: 911-915. 


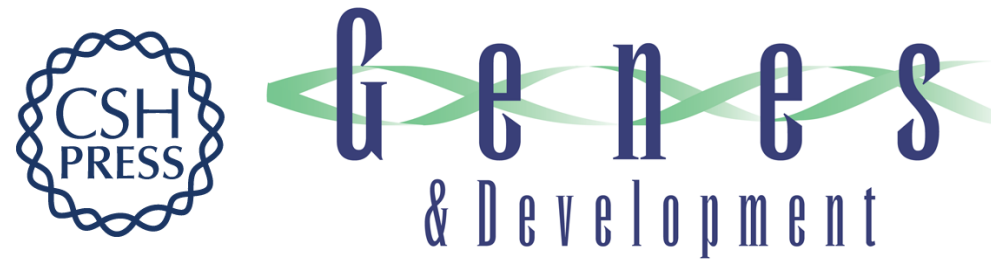

\section{An avian muscle factor related to MyoD1 activates muscle-specific promoters in nonmuscle cells of different germ-layer origin and in BrdU-treated myoblasts.}

Z Y Lin, C A Dechesne, J Eldridge, et al.

Genes Dev. 1989, 3:

Access the most recent version at doi:10.1101/gad.3.7.986

References This article cites 36 articles, 13 of which can be accessed free at: http://genesdev.cshlp.org/content/3/7/986.full.html\#ref-list-1

License

Email Alerting Service

Receive free email alerts when new articles cite this article - sign up in the box at the top right corner of the article or click here.

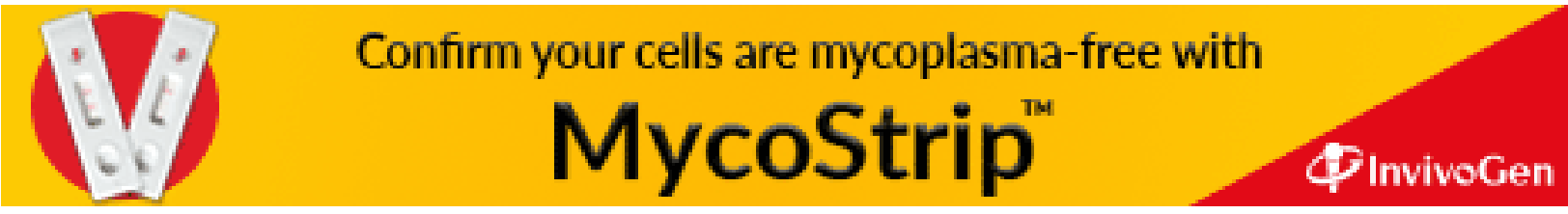

